\title{
OPTIMAL APPROXIMATION, SIMULATION AND ANALOG REALIZATION OF THE FUNDAMENTAL FRACTIONAL ORDER TRANSFER FUNCTION
}

\author{
Abdelbaki DJOUAmbi *, Abdelfatah CHAREF **, Alina Voda BeSAnÇON *** \\ * Department of Electronics, University of Oum El-Bouaghi \\ Oum El-Bouaghi 04000, Algeria \\ e-mail: djouambi_abdelbaki@yahoo.fr \\ ** Department of Electronics, University Mentouri of Constantine \\ Route Ain El-Bey, Constantine 25000, Algeria \\ e-mail: afcharef@yahoo.com \\ *** Laboratory of Automatic Control of Grenoble, LAG-ENSIEG \\ BP 46 Rue de la Houille Blanche St Martin d'Hères 38402 \\ France \\ e-mail: alina.voda@lag.ensieg.inpg.fr
}

\begin{abstract}
This paper provides an optimal approximation of the fundamental linear fractional order transfer function using a distribution of the relaxation time function. Simple methods, useful in systems and control theories, which can be used to approximate the irrational transfer function of a class of fractional systems for a given frequency band by a rational function are presented. The optimal parameters of the approximated model are obtained by minimizing simultaneously the gain and the phase error between the irrational transfer function and its rational approximation. A simple analog circuit which can serve as a fundamental analog fractional system is obtained. Illustrative examples are presented to show the quality and usefulness of the approximation method.
\end{abstract}

Keywords: fractional systems, approximation, simulation and distribution of the relaxation times function

\section{Introduction}

Since the first work on fractional order derivatives published several centuries ago, the theory of fractional calculus has been mostly developed by mathematicians. In the last decades a considerable focus on fractional calculus has been stimulated by applications of these concepts in various areas of physics and engineering. Many systems are known to reveal fractional order system dynamics, such as viscoelastic systems (Torvik and Bagley, 1984), electrode-electrolyte polarization (Ichise et al., 1971), interface polarization (Sun and Onaral, 1983), cardiac behaviour (Goldberger et al., 1985), dielectric relaxation (Cole and Cole, 1941; Davidson and Cole, 1950). Because of their representation by irrational transfer functions, fractional order systems were studied marginally in theory and practice. But a great effort was made to put into practice some established results. It is only in recent years that one can find significant progress in theoretical works which serve as a foundation for an increasing number of applications in systems and control fields (Manabe, 1961; Oustaloup, 1983; Charef et al., 1992; Miller and Ross, 1993; Podlubny, 1994; 1999; Hartley and Lorenzo, 1998; Petras et al., 2002). However, intensive research work is still under way in many electrical engineering areas towards the application of these fractional order concepts.

The fundamental linear fractional order transfer function commonly used to describe some typical fractional systems, defined in (Hartley and Lorenzo, 1998), is represented by the following equation:

$$
H(s)=\frac{b_{0}}{1+\left(\tau_{0} s\right)^{m}},
$$

where $b_{0}, \tau_{0}$ and $m$ are positive real numbers. This type of expression gives a much more suitable mathematical representation to the natural phenomena.

Transfer functions such as (1) are not easy to implement for computational purposes. Since their mathemati- 
cal representations in the frequency domain are irrational, direct analysis methods and corresponding time domain behavior seem difficult to handle. As for the identification, analysis, synthesis and simulation of the corresponding systems, a need arises for a rational function approximation.

This makes the task of finding integer order approximations of fractional transfer functions a most important one. What is meant by this is that when simulations are to be performed or models are to be identified or controllers are to be implemented, fractional transfer functions, are usually replaced by rational transfer functions which are easier to handle. During the last twenty years numerical algorithms have been developed to approximate fractional order systems using either continuous or discrete rational models approximating, see (Vinager et al., 2000; Chen and Moore, 2002; Aoun et al., 2003; Poinot and Trigeassou 2004; Barbosa et al. 2006). It is not possible to say that one of them is the best, because even though some are better then others with regard to certain characteristics, the relative merits of each approximation depend on the fractional powers, on whether one is more interested in an accurate frequency behavior or in accurate time responses, on how large admissible transfer functions may be, and other factors like these.

In this paper new simple methods, very useful in systems and control theories, are presented to approximate the fundamental fractional order transfer function given in (1) by a rational function. A distribution of the relaxation time function is used. The optimal distribution of the relaxation time is obtained by minimizing simultaneously the gain and the phase error between the original fractional system and its rational approximation. Section 2 summarizes the optimal approximation, simulation and analog realization of the relaxation fractional system represented by the Cole-Cole transfer function for $0<m<1$. In Section 3 we present the approximation and analog realization of the oscillation fractional system given by (1) for $1<m<2$. Illustrative examples are presented to show the quality and usefulness of the proposed approach.

\section{Relaxation Fractional Order System}

2.1. Definition. A relaxation fractional system is defined in this context by the following fundamental linear fractional order differential equation for $0<m<1$ :

$$
\left(\tau_{0}\right)^{m} \frac{\mathrm{d}^{m} y(t)}{\mathrm{d} t^{m}}+y(t)=e(t)
$$

Its transfer function is given by

$$
H(s)=\frac{1}{1+\left(\tau_{0} s\right)^{m}},
$$

where $\tau_{0}$ is a positive real number.
2.2. Rational Function Approximation. In dielectric studies, Cole and Cole (1941) observed that dispersion/relaxation data measured from a large number of materials can be modeled by the function (3), where $m$ is a real number such that $0<m<1$. It is also known that the distribution of the relaxation times function $G(\tau)$ can be derived directly from the original transfer function as (Fuross and Kirkwood, 1941):

$$
H(s)=\int_{0}^{\infty} \frac{G(\tau)}{1+\tau s} \mathrm{~d} \tau .
$$

Cole and Cole (1941) applied this method to find the distribution function $G(\tau)$ for the model (3), which gives

$$
G(\tau)=\frac{1}{2 \pi} \frac{\sin [(1-m) \pi]}{\cosh \left[m \log \left(\frac{\tau}{\tau_{0}}\right)\right]-\cos [(1-m) \pi]} .
$$

The proposed approximation method starts with sampling the distribution of the relaxation times function $G(\tau)$ of Eqn. (5) for a limited frequency band of practical interest $\left[w_{\min }, w_{\max }\right]$ at logarithmically equidistant points $\tau_{i}$ (Sun et al., 1992):

$$
G(\tau)=\sum_{i=1}^{2 N-1} G\left(\tau_{i}\right) \delta\left(\tau-\tau_{i}\right)
$$

where

$$
\tau_{i}=\tau_{0}(\lambda)^{(N-i)}, \quad i=1,2, \ldots, 2 N-1
$$

are the relaxation times and $\lambda>1$ is the ratio of a time constant to the next one or the ratio of a pole to the previous one, where the poles are given by

$$
p_{i}=1 / \tau_{i}, \quad i=1,2, \ldots, 2 N-1 .
$$

Then the transfer function of Eqn. (3) can be approximated by

$$
H(s) \approx H_{N}(s)=b \sum_{i=1}^{2 N-1} \frac{G\left(\tau_{i}\right)}{1+\tau_{i} s},
$$

where $b$ is a positive real number.

From the condition

$$
\left.H(j w) \approx H_{N}(j w)\right|_{w=w_{\min }},
$$

the parameter $b$ can be defined by the following equation:

$$
b=\frac{\left|H\left(j w_{\min }\right)\right|}{\left|\sum_{i=1}^{2 N-1} \frac{G\left(\tau_{i}\right)}{1+\tau_{i} j w_{\min }}\right|} .
$$

In almost all cases the frequency $w_{\min }$ is supposed to be close to zero $\left(w_{\min } \approx 0\right)$. Thus, the previous formulas simplify to

$$
b=\frac{1}{\sum_{i=1}^{2 N-1} G\left(\tau_{i}\right)}
$$


For a given frequency band $w_{\max }$ the number $N$ is obtained from the following equation (Sun et al., 1992):

$$
N=\text { Integer }\left[\frac{\log \left(\tau_{0} w_{\max }\right)}{\log (\lambda)}\right]+1
$$

For given values of $N$ and $m$, the optimal ratio $\lambda_{o p}$ can be calculated by minimizing the criterion $J(\lambda)$ given by the following equation within the frequency band of interest $w \in\left[w_{\min }, w_{\max }\right] \mathrm{rd} / \mathrm{s}$ :

$$
\begin{aligned}
J(\lambda)= & \sum_{w_{\min }}^{w_{\max }}\left[\alpha\left(\left|H_{N}(j w)\right|-|H(j w)|\right)^{2}\right. \\
& +(1-\alpha) \arg \left(H_{N}(j w)-\arg (H(j w))^{2}\right],
\end{aligned}
$$

where $0<\alpha<1$ is the scaling ratio. We have

$$
\left[\lambda_{o p}\right]=\arg \min (J(\lambda))
$$

This is a nonlinear optimization problem, which justifies a recourse to nonlinear optimization methods. Using a genetic algorithm implemented in Matlab, the optimal values of the ratio $\lambda$ for a given scaling ratio $\alpha=0.5$ and for different values of $m$ and $N$ are given in Table 1 .

Table 1. Optimal ratio $\lambda_{o p}$, the maximum gain error $\left(\max _{e g}\right)$ and the maximum phase error $\max _{e p h}$ obtained using the proposed approximation method for different values of $m$ and $N$, with $\tau_{0}=1$.

\begin{tabular}{|c|c|c|c|c|}
\hline & & $N=10$ & $N=20$ & $N=40$ \\
\hline \hline \multirow{3}{*}{$m=0.1$} & $\lambda_{o p}$ & 22.644 & 10.343 & 4.748 \\
\cline { 2 - 5 } & $\left|\mathrm{max}_{e g}\right|_{\mathrm{dB}}$ & 1.162 & 0.233 & 0.052 \\
\cline { 2 - 5 }$m=0.4$ & $\left|\max _{e p h}\right|_{\mathrm{deg}}$ & 2.725 & 0.644 & 0.105 \\
\hline \multirow{3}{*}{$m=0.7$} & $\lambda_{o p}$ & 7.115 & 3.645 & 2.640 \\
\cline { 2 - 5 } & $\left|\max _{e g}\right|_{\mathrm{dB}}$ & 0.331 & 0.028 & 0.002 \\
\cline { 2 - 5 } & $\left|\max _{e p h}\right|_{\mathrm{deg}}$ & 1.610 & 0.125 & 0.004 \\
\hline \multirow{3}{*}{$m=0.9$} & $\lambda_{o p}$ & 5.044 & 2.740 & 2.261 \\
\cline { 2 - 5 } & $\left|\max _{e g}\right|_{\mathrm{dB}}$ & 0.225 & 0.013 & 0.004 \\
\hline & $\left|\max _{e p h}\right|_{\mathrm{deg}}$ & 1.680 & 0.092 & 0.014 \\
\cline { 2 - 5 } & $\lambda_{o p}$ & 3.563 & 1.987 & 1.488 \\
\cline { 2 - 5 } & $\left|\max _{e p h}\right|_{\mathrm{dB}}$ & 2.451 & 0.599 & 0.056 \\
\hline
\end{tabular}

2.3. Time Response. From (9) we have

$$
H(s)=\frac{Y(s)}{E(s)}=\frac{1}{1+\left(\tau_{0} s\right)^{m}} \cong b \sum_{i=1}^{2 N-1} \frac{G\left(\tau_{i}\right)}{1+\tau_{i} s} .
$$

For $e(t)=\delta(t)$ (a unit impulse), we get

$$
Y(s)=b \sum_{i=1}^{2 N-1} \frac{G\left(\tau_{i}\right)}{1+\tau_{i} s} .
$$

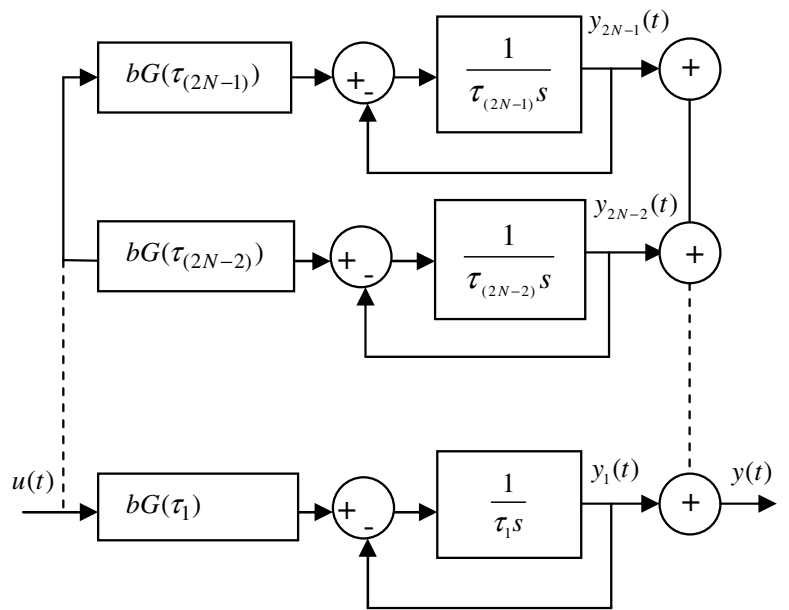

Fig. 1. Simulation scheme of the fractional system $H(s)=1 /\left(1+\left(\tau_{0} s\right)^{m}\right)$ for $0<m<1$.

Using the inverse Laplace transform, we deduce the impulse response of (16):

$$
y(t)=b \sum_{i=1}^{2 N-1} \frac{G\left(\tau_{i}\right)}{\tau_{i}} \exp \left(-\frac{t}{\tau_{i}}\right) .
$$

Now, for $e(t)=u(t)$ (a unit step), we have

$$
\begin{aligned}
Y(s) & =b \sum_{i=1}^{2 N-1} \frac{G\left(\tau_{i}\right)}{1+\tau_{i} s} \frac{1}{s} \\
& =b \sum_{i=1}^{2 N-1} G\left(\tau_{i}\right)\left(\frac{1}{s}-\frac{\tau_{i}}{1+\tau_{i} s}\right) .
\end{aligned}
$$

Using the inverse Laplace transform, we get the step response of (18):

$$
y(t)=b \sum_{i=1}^{2 N-1} G\left(\tau_{i}\right)\left(1-\exp \left(-\frac{t}{\tau_{i}}\right)\right) .
$$

Figure 1 provides the simulation scheme for the discussed system for $0<m<1$.

2.4. Analog Circuit Realization. It can be easily seen that (15) is analogous to the impedance $Z(s)$ of $2 N-1$ parallel RC cells connected in series as shown in Fig. 2:

$$
Z(s)=\sum_{i=1}^{2 N-1} \frac{R_{i}}{1+s R_{i} C_{i}} .
$$

From (15) and (20), and for $i=1,2, \ldots, 2 N-1$, we can write

$$
R_{i}=b G\left(\tau_{i}\right), \quad R_{i} C_{i}=\tau_{i} .
$$

Hence the resistor and capacitor parameters of the analog circuit modeling the simple fractional order system in a given frequency band are

$$
R_{i}=b G\left(\tau_{i}\right), \quad C_{i}=\frac{\tau_{i}}{b G\left(\tau_{i}\right)},
$$

$i=1,2, \ldots, 2 N-1$. 


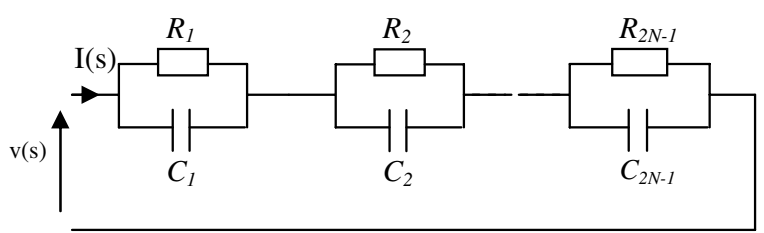

Fig. 2. Analog RC circuit realization of the fundamental fractional order system, $0<m<1$.

2.5. Illustrative Example. For illustration, consider a numerical example for a relaxation fractional order system represented by the Cole-Cole transfer function given by the following equation with $m=0.7$ and $\tau_{0}=1$ :

$$
H(s)=\frac{1}{1+s^{0.7}} .
$$

For a frequency band of practical interest $\left[w_{\min }, w_{\max }\right]=$ $\left[10^{-3}, 10^{3}\right] \mathrm{rad} / \mathrm{s}$, and a given number $N=10$, the optimal ratio $\lambda_{o p}$, the relaxation times $\tau_{i}$, the factor $b$ and the distribution $G\left(\tau_{i}\right)$ for a given scaling ratio $\alpha=0.5$ can be easily calculated based on the results of Section 2.2:

$$
\begin{aligned}
\lambda_{\text {op }} & =5.044, \quad \tau_{0}=1, \quad \tau_{i}=(5.044)^{(10-i)}, \\
G\left(\tau_{i}\right) & =\frac{1}{2 \pi} \frac{\sin [0.3 \pi]}{\cosh [0.7(10-i) \log (5.044)]-\cos [0.3 \pi]}, \\
b & =\frac{1}{\sum_{i=1}^{19} G\left(\tau_{i}\right)}, \quad i=1,2, \ldots, 19 .
\end{aligned}
$$

Figures 3 and 4 show the Bode plots of the relaxation fractional order system and its proposed rational function approximation along with the rational function approximation by Oustaloup's method. Figures 5 and 6 show the magnitude and phase errors of the proposed method and those of Oustaloup's approximations (Oustaloup, 1995). One can easily see that the proposed method yields a satisfactory approximation over the entire frequency band of interest.

Figure 7 additionally shows the step responses of this fractional order system obtained from the proposed rational function approximation and from the rational function approximations by the Oustaloup and Grünwalds methods given in (Oustaloup, 1995).

\section{Oscillatory Fractional Order System}

3.1. Definition. An oscillatory fractional order system is defined in this context as a fundamental linear fractional order differential equation (3) for $1<m<2$.

3.2. Rational Function Approximation. The transfer function of the oscillatory fractional order system given by (3) can be modeled by the following function:

$$
\begin{aligned}
H(s) & =\frac{1}{1+\left(\tau_{0} s\right)^{m}} \approx \frac{\left(\tau_{0} s+1\right)^{(2-m)}}{\left(\tau_{0} s\right)^{2}+2 \xi \tau_{0} s+1} \\
& =H_{N}(s) H_{D}(s),
\end{aligned}
$$

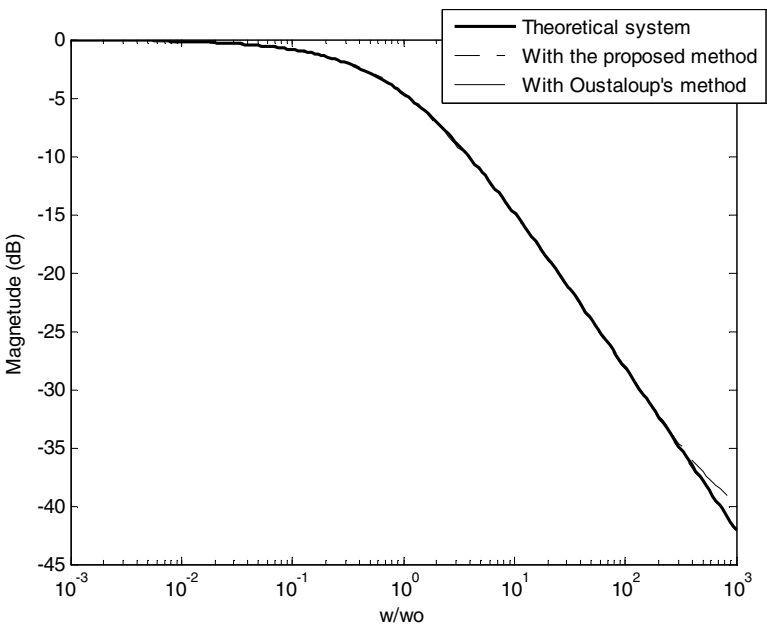

Fig. 3. Magnitude Bode plot of $H(s)=1 /\left(1+s^{0.7}\right)$ of the proposed rational function approximation and of the rational function approximated by Oustaloup's method.

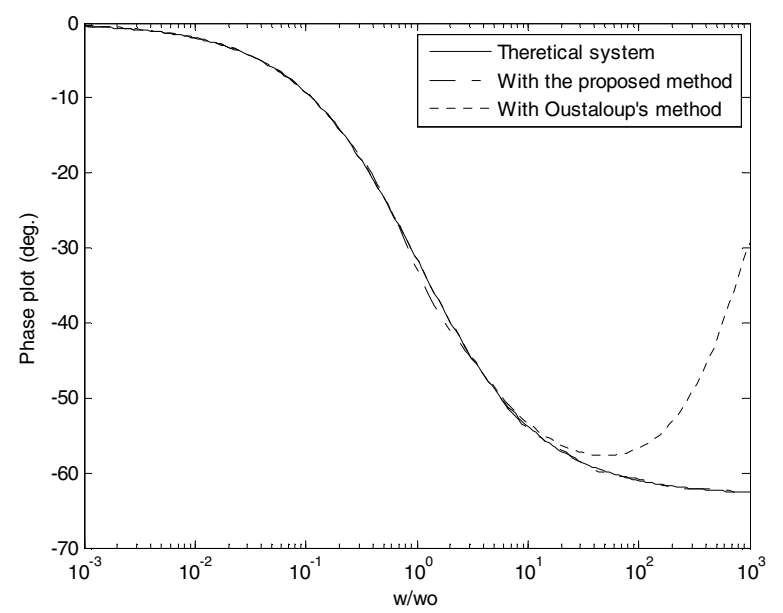

Fig. 4. Phase Bode plot of $H(s)=1 /\left(1+s^{0.7}\right)$ of the proposed rational function approximation and of the rational function approximated by Oustaloup's method.

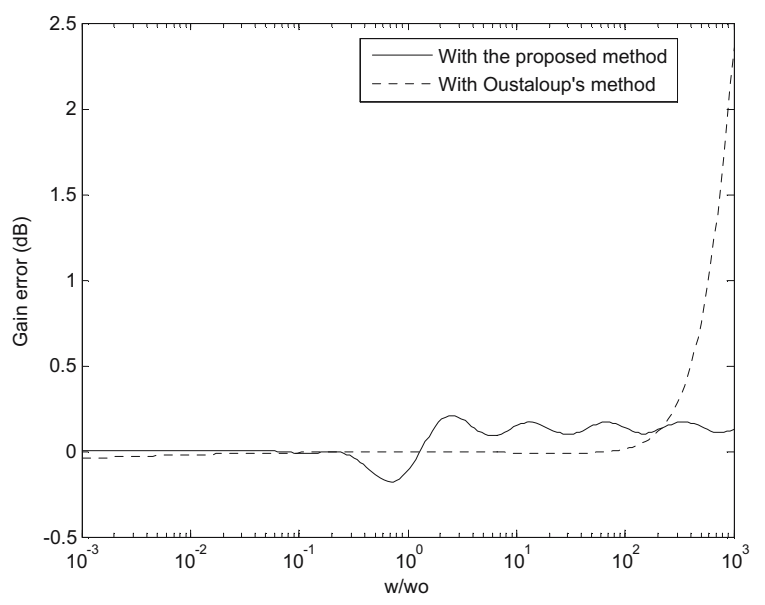

(a)

Fig. 5. Magnitude error plots of the approximation of $H(s)=$ $1 /\left(1+s^{0.7}\right)$ using the proposed and Oustaloup methods. 


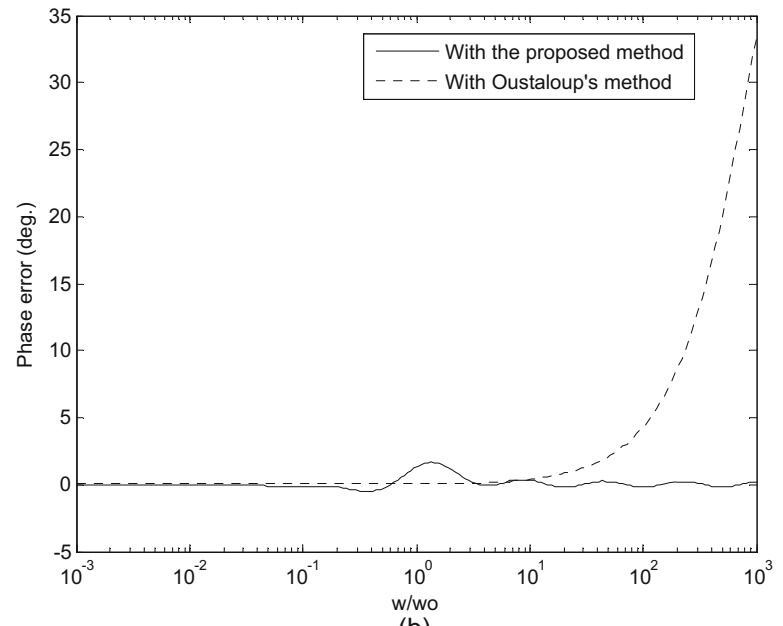

(b)

Fig. 6. Phase error plots of the approximations of $H(s)=$ $1 /\left(1+s^{0.7}\right)$ using the proposed and Oustaloup methods.

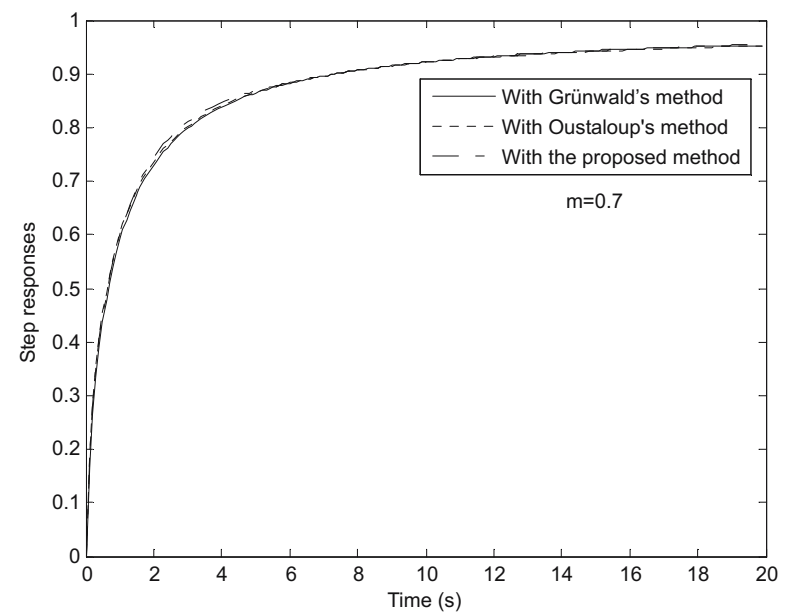

Fig. 7. Step responses of $H(s)=1 /\left(1+s^{0.7}\right)$ using the proposed rational function approximation, the rational function approximated by Oustaloup's method and Grünwald's method.

where

$$
H_{N}(s)=\left(\tau_{0} s+1\right)^{(2-m)}
$$

represents the Fractional Power Zero (FPZ) with $0<(2-$ $m)<1$, and

$$
H_{D}(s)=\frac{1}{\left(\tau_{0} s\right)^{2}+2 \xi \tau_{0} s+1}
$$

is a regular second-order system. It can be easily shown that for $|H(j w)| \cong\left|H_{N}(j w)\right|\left|H_{D}(j w)\right| \cong 1, w \ll 1 / \tau_{0}$ for $|H(j w)| \cong\left|H_{N}(j w)\right|\left|H_{D}(j w)\right| \cong \frac{1}{\left(\tau_{0} w\right)^{m}}, w \gg$ $1 / \tau_{0}$ and for $w=1 / \tau_{0}$ we get

$$
|H(j w)|=\left|\frac{1}{1+(j)^{m}}\right|=\frac{1}{\sqrt{\left(1+\cos m \frac{\pi}{2}\right)^{2}+\left(\sin m \frac{\pi}{2}\right)^{2}}}
$$

$$
\cong\left|\frac{(j+1)^{(2-m)}}{2 \xi j}\right|=\frac{(\sqrt{2})^{2-m}}{2 \xi} .
$$

From (26), the damping ratio $\xi$ of a regular secondorder system must be given as

$$
\xi=\frac{\sqrt{(1+\cos (m \pi / 2)})}{(\sqrt{2})^{m-1}} .
$$

To represent the oscillatory fractional order system of (23) by a linear time-invariant system model, it is necessary to approximate it, for a limited frequency band of practical interest $\left[0, w_{\max }\right]$, by a rational function. To this end, we have to approximate the fractional power zero of (24) by a rational function in the frequency band of practical interest $\left[0, w_{\max }\right]$. The proposed method consists in approximating its $20(2-m) \mathrm{dB} / \mathrm{dec}$ slope on the Bode plot by a number of zig-zag lines with alternate slopes of $20 \mathrm{~dB} / \mathrm{dec}$ and $0 \mathrm{~dB} /$ dec corresponding to alternate zeros and poles on the negative real axis of the $s$-plane such that $z_{0}<p_{0}<z_{1}<p_{1}<\cdots<z_{n}<p_{n}$. Hence we can write

$$
H_{N}(s)=\left(\tau_{0} s+1\right)^{(2-m)} \cong \frac{\prod_{i=0}^{N}\left(1+\frac{s}{z_{i}}\right)}{\prod_{i=0}^{N}\left(1+\frac{s}{p_{i}}\right)} .
$$

Accordingly, Eqn. (23) can be rewritten as

$$
\begin{aligned}
H(s) & =\frac{1}{1+\left(\tau_{0} s\right)^{m}} \\
& \approx\left[\frac{1}{\left(\tau_{0} s\right)^{2}+2 \xi \tau_{0} s+1}\right] \frac{\prod_{i=0}^{N}\left(1+\frac{s}{z_{i}}\right)}{\prod_{i=0}^{N}\left(1+\frac{s}{p_{i}}\right)} .
\end{aligned}
$$

The same idea was used to approximate the Fractional Power Pole (FPP) given in (Charef et al., 1992). Using a simple graphical method, the zeros $z_{i}$ and the poles $p_{i}$ of the approximation for a specified error $\varepsilon$ in $\mathrm{dB}$ were found to be in a geometric progression form:

$$
\begin{aligned}
H(s) & =\frac{1}{1+\left(\tau_{0} s\right)^{m}} \\
& \approx\left[\frac{1}{\left(\tau_{0} s\right)^{2}+2 \xi \tau_{0} s+1}\right] \frac{\prod_{i=0}^{N}\left(1+\frac{s}{z_{0}(a b)^{i}}\right)}{\prod_{i=0}^{N}\left(1+\frac{s}{p_{0}(a b)^{i}}\right)},
\end{aligned}
$$

where

$$
\begin{aligned}
a & =10^{\left[\frac{\varepsilon}{10(m-1)}\right]} \quad b=10^{\left[\frac{\varepsilon}{10(2-m)}\right]}, \\
z_{0} & =\frac{1}{\tau_{0}} \sqrt{b}, \quad p_{0}=a z_{0} \\
N & =\text { Integer }\left[\frac{\log \frac{w_{\max }}{z_{0}}}{\log (a b)}\right]+1 .
\end{aligned}
$$


3.3. Time Responses. By partial fraction expansion of the rational function (30), it is possible to represent the transfer function of the oscillatory fractional order system by a linear combination of elementary simple functions, i.e.,

$$
\begin{aligned}
H(s) & =\frac{1}{1+\left(\tau_{0} s\right)^{m}} \\
& \approx \frac{A s+B}{\left(\tau_{0} s\right)^{2}+2 \xi \tau_{0} s+1}+\sum_{i=0}^{N} \frac{k_{i}}{1+\frac{s}{p_{0}(a b)^{i}}},
\end{aligned}
$$

where $k_{i}, i=0,1, \ldots, N$ are the residues of the poles,

$$
\begin{aligned}
k_{i}= & {\left[\frac{1}{\left(\tau_{0} p_{0}(a b)^{i}\right)^{2}-2 \xi \tau_{0} p_{0}(a b)^{i}+1}\right] } \\
& \times \frac{\prod_{j=0}^{N}\left[1-a(a b)^{(i-j)}\right]}{\prod_{\substack{j=0 \\
j \neq i}}^{N}\left[1-(a b)^{(i-j)}\right]} .
\end{aligned}
$$

The constants $A$ and $B$ can be calculated as

$$
B=1-\sum_{i=0}^{N} k_{i}, \quad A=-\tau_{0}^{2} \sum_{i=0}^{N} k_{i} p_{0}(a b)^{i} .
$$

Consequently,

$$
\begin{aligned}
H(s) & =\frac{Y(s)}{E(s)} \\
& =\frac{A s+B}{\left(\tau_{0} s\right)^{2}+2 \xi \tau_{0} s+1}+\sum_{i=0}^{N} \frac{k_{i}}{1+\frac{s}{p_{0}(a b)^{i}}} .
\end{aligned}
$$

Using the inverse Laplace transform, the step response of (33) can be obtained as

$$
\begin{aligned}
y(t)=1 & -\sum_{i=0}^{N} k_{i} \exp \left(-p_{0}(a b)^{i} t\right) \\
& +C \exp \left(-\frac{\xi}{\tau_{0}} t\right) \sin \left(\frac{\sqrt{1-\xi^{2}}}{\tau_{0}}+\phi\right)
\end{aligned}
$$

where the constants $C$ and $\phi$ are given as follows (Kuo, 1995):

$$
C=B \sqrt{\frac{A^{2}-2 A B \xi \tau_{0}+\left(B \tau_{0}\right)^{2}}{\left(B \tau_{0}\right)^{2}\left(1-\xi^{2}\right)}}
$$

and

$\phi=\arctan \left(\frac{A \sqrt{1-\xi^{2}}}{B \tau_{0}-A \xi}\right)-\arctan \left(\frac{\sqrt{1-\xi^{2}}}{-\xi}\right)$.
3.4. Analog Circuit Realization. From (30), the transfer function of the oscillation fractional order system defined above is given by

$$
\begin{aligned}
H(s) & =\frac{1}{1+\left(\tau_{0} s\right)^{m}} \\
& \approx\left[\frac{1}{\left(\tau_{0} s\right)^{2}+2 \xi \tau_{0} s+1}\right] \frac{\prod_{i=0}^{N}\left(1+\frac{s}{z_{0}(a b)^{i}}\right)}{\prod_{i=0}^{N}\left(1+\frac{s}{p_{0}(a b)^{i}}\right)} \\
& =H_{1}(s) H_{2}(s) .
\end{aligned}
$$

The above transfer function can be realized by the analog circuit of Fig. (6) as

$$
\begin{aligned}
H(s)= & \frac{Y(s)}{E(s)}=\left(\frac{1}{L s}\right)\left(\frac{L s}{L C s^{2}+\frac{L}{R} s+1}\right) \\
& \times\left(\frac{1}{R_{p}}+\sum_{i=0}^{N} \frac{C_{i} s}{1+R_{i} C_{i} s}\right)\left(R_{S}\right),
\end{aligned}
$$

where $R_{S}=R_{p}=1 \Omega, L=2 R \xi \tau_{0}, C=\tau_{0} / 2 R \xi$,

$$
\begin{aligned}
C_{i} & =\frac{1}{p_{0}(a b)^{i}} \frac{\prod_{j=0}^{N}\left(1-a(a b)^{i-j}\right)}{\prod\left(1-(a b)^{i-j}\right)} \\
R_{i} & =\frac{1}{p_{0}(a b)^{i} C_{i}} .
\end{aligned}
$$

3.5. Illustrative Example. This section presents simulation results for an oscillation fractional order system represented by the following fractional order transfer function:

$$
H(s)=\frac{1}{1+s^{1.6}} .
$$

First, $H(s)$ is modeled by the following function:

$$
H(s)=\frac{1}{1+s^{1.6}} \approx \frac{(s+1)^{0.4}}{s^{2}+(0.71) s+1} .
$$

For a frequency band of practical interest $\left[10^{-3}, 10^{+3}\right] \mathrm{rad} / \mathrm{s}$ and for an approximation error $\varepsilon=1 \mathrm{~dB}$, the approximation of the fractional power zero $(1+s)^{0.4}$ by a rational function can be given by

$$
(s+1)^{0.4}=\frac{\prod_{i=0}^{N}\left(1+\frac{s}{z_{0}(a b)^{i}}\right)}{\prod_{i=0}^{N}\left(1+\frac{s}{p_{0}(a b)^{i}}\right)},
$$

where the parameters $a, b, z_{0}, p_{0}$ and $N$ can be easily calculated as follows:

$$
\begin{aligned}
a & =1.468, \quad b=1.778, \quad p_{0}=1.957 \mathrm{rad} / \mathrm{s}, \\
z_{0} & =1.334 \mathrm{rad} / \mathrm{s}, \quad N=8 .
\end{aligned}
$$




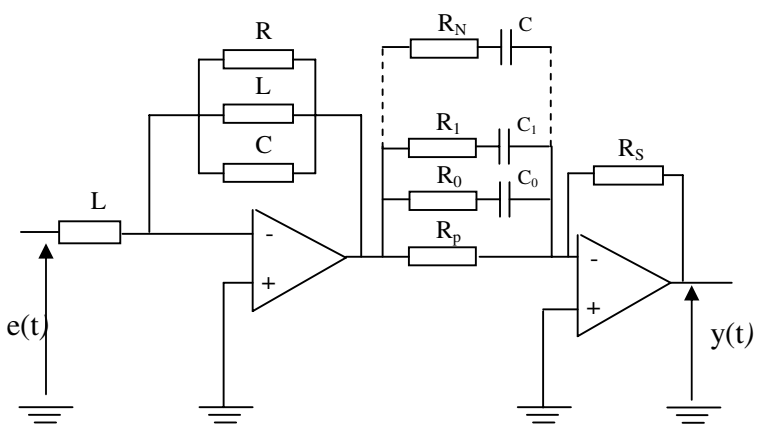

Fig. 8. Analog RLC circuit realization of the fundamental fractional order system for $1<m<2$.

Then we have

$$
\begin{aligned}
H(s) & =\frac{1}{1+s^{1.6}} \\
& \approx\left[\frac{1}{s^{2}+(0.71) s+1}\right] \frac{\prod_{i=0}^{8}\left(1+\frac{s}{1.334(2.61)^{i}}\right)}{\prod_{i=0}^{8}\left(1+\frac{s}{1.957(2.61)^{i}}\right)} .
\end{aligned}
$$

Figures 9 and 10 show the Bode plots of the relax-

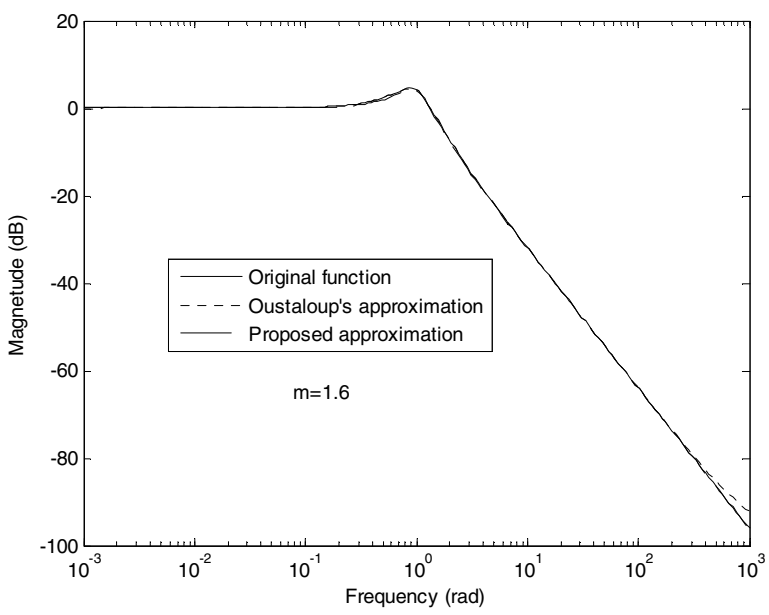

Fig. 9. Magnitude Bode plot of $H(s)=1 /\left(1+s^{1.6}\right)$ of the proposed rational function approximation and of the rational function approximated by Oustaloup's method.

ation fractional order system transfer function given above and its proposed rational function approximation along with the rational function approximation by Oustaloup's method. We can easily see that the Bode plots of the proposed method and the original function are all quite overlapping over the frequency band of interest.

Figure 11 shows the step responses of this fractional order system obtained from its proposed rational function approximation, from the rational function approximation by Oustaloup's method and by the Grünwald method given in (Oustaloup, 1995).

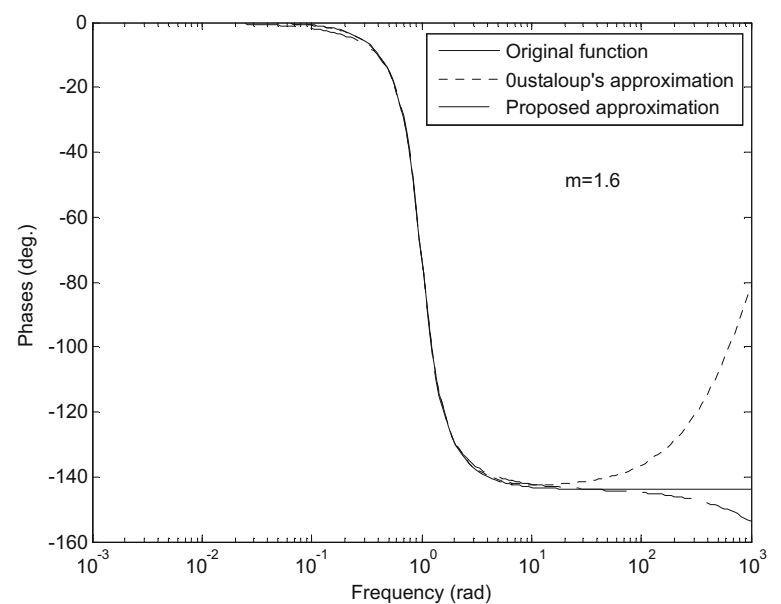

Fig. 10. Phase Bode plot of $H(s)=1 /\left(1+s^{1.6}\right)$ of the proposed rational function approximation and of the rational function approximated by Oustaloup's method.

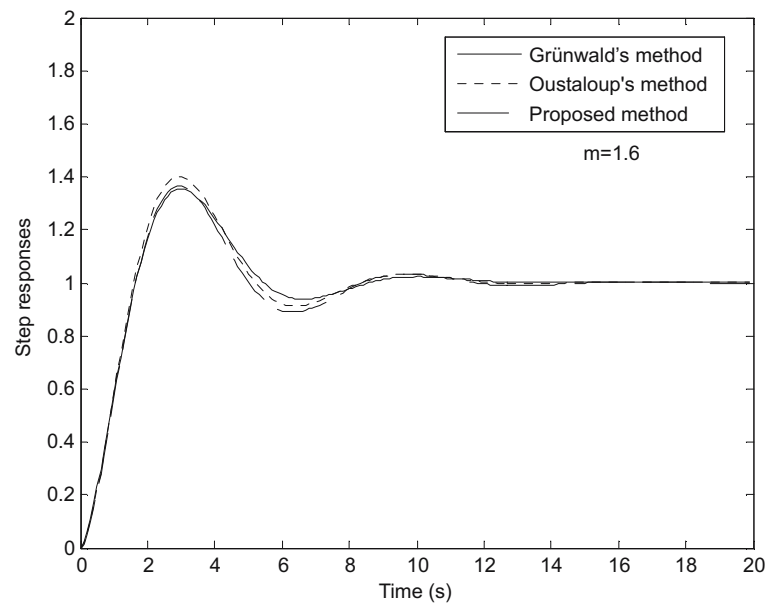

Fig. 11. Step responses of $H(s)=1 /\left(1+s^{1.6}\right)$ using the proposed rational function approximation, the rational function approximated by Oustaloup's method and Grünwalds method.

\section{Conclusion}

In this paper we presented some effective methods, very useful in systems and control theories, for optimal approximation of the irrational function given by $H(s)=$ $1 /\left(1+\left(\tau_{0} s\right)^{m}\right)$ for $0<m<2$ representing the fundamental fractional order transfer function, by a rational function in a given frequency band of practical interest. Using this approximation, the step responses of this type of system are derived and simple analog circuit realizations are also obtained. Illustrative examples demonstrated the usefulness of the proposed methods. This approximation is very suitable for simulation, modeling and parameter identification of a class of fractional systems and useful in the analysis, realization and implementation of fractional order control systems because it is possible 
to do the analysis and the design directly in the $s$-plane.

\section{References}

Aoun M., Malti R., Levron F and Oustaloup A. (2003): Numerical simulation of fractional systems. Proceedings of DETC'03 ASME 2003 Design Engineering Technical Conference and Computers and Information in Engineering Conference, Chicago, USA.

Barbosa R.S., Machado T.J.A. and Silva M.F. (2006): Descritization of complex-order differintegrals. Proceedings of the 2nd IFAC Workshop on Fractional Differentiation and its Applications, Porto, Portugal, pp. 340-345.

Cole K.S. and Cole R.H. (1941): Dispersion and absorption in dielectrics, alternation current characterization. Journal of Chemical Physics Vol. 9, pp. 341-351.

Charef A., Sun H.H., Tsao Y.Y. and Onaral B. (1992): Fractal system as represented by singularity function. IEEE Transactions on Automatic Control, Vol. 37, No. 9, pp. 14651470.

Chen Y.Q. and Moore K.L. (2002): Discretization schemes for fractional-order differentiators and integrators. IEEE Transactions on Circuits and Systems I: Fundamental Theory and Applications, Vol. 49, No. 3, pp. 363-367.

Davidson D. and Cole R. (1950), Dielectric relaxation in glycerine. Journal of Chemical Physics, Vol. 18, pp. 1417-1418.

Fuross R.M. and Kirkwood J.K. (1941): Electrical properties of solids VIII-Dipole moments in polyvinyl chloride biphenyl systems. Journal of the American Chemical Society, Vol. 63, pp. 385-394.

Hartley T.T. and Lorenzo C.F. (1998): A solution of the fundamental linear fractional order differential equation. Technical Report No. TP-1998-208693, NASA, Ohio.

Goldberger A.L., Bhargava V., West B.J. and Mandell A.J. (1985): On the mechanism of cardiac electrical stability. Biophysics Journal, Vol. 48, pp. 525-528.

Ichise M., Nagayanagi Y and Kojima T. (1971): An analog simulation of non-integer order transfer functions for analysis of electrode processes. Journal of Electro-Analytical Chemistry, Vol. 33, pp. 253-265.

Kuo, Benjamin C. (1995): Automatic Control Systems. Englewood Cliffs: Prentice-Hall.
Manabe S. (1961): The non-integer integral and its application to control systems. ETJ of Japan, Vol. 6, Nos. 3-4, pp. 8387.

Miller K.S. and Ross B. (1993): An Introduction to the Fractional Calculus and Fractional Differential Equations. New-York: Wiley.

Oustaloup A. (1983) : Systèmes Asservis Linéaires d'Ordre Fractionnaire: Théorie et Pratique. Paris: Masson.

Oustaloup A. (1995) : La Dérivation Non Entière, Théorie, Synthèse et Application. Paris: Hermes.

Poinot T. and Trigeassou J. C. (2004): Modelling and simulation of fractional systems. Proceedings of the 1st IFAC Workshop on Fractional Differentiation and its Application, Bordeaux, France, pp. 656-663.

Podlubny I. (1994): Fractional-order systems and fractionalorder controllers. Technical Report No. UEF-03-94, Slovak Academy of Sciences, Košice Slovakia.

Podlubny I. (1999): Fractional Differential Equations. San Diego: Academic Press.

Petras I., Podlubny I., Vinagre M.,Dorcak L. and O'Learya P. (2002): Analogue Realization of Fractional Order Controllers. Fakulta Berg, Technical University of Košice, Slovakia.

Sun H.H. and Onaral B. (1983): A unified approach to represent metal electrode polarization. IEEE Transactions on Biomedical Engineering, Vol. 30, pp. 399-406.

Sun H.H., Charef A., Tsao Y.Y. and Onaral B. (1992): Analysis of polarization dynamics by singularity decomposition method. Annals of Biomedical Engineering, Vol. 20, pp. 321-335.

Torvik P.J. and Bagley R.L. (1984): On the appearance of the fractional derivative in the behavior of real materials. Transactions of the ASME, Vol. 51, pp. 294-298.

Vinagere B.M., Podlubny I., Hernandez A. and Feliu V. (2000): Some approximations of fractional order operators used in control theory and applications. Journal of Fractional Calculus and Applied Analysis, Vol. 3, No. 3, pp. 231-248.

Received: 6 April 2007

Revised: 12 September 2007

Re-revised: 15 December 2006 